\title{
基于水解-沉淀形态分布的金属盐混凝过程关键 因子解析
}

\author{
甘永海, 张丽, 吴兵党, 郑宏岑, 张淑娟 ${ }^{*}$ \\ 南京大学环境学院, 污染控制与资源化研究国家重点实验室, 南京 210023 \\ *通讯作者, E-mail: sjzhang@nju.edu.cn \\ 收稿日期: 2020-12-04; 接受日期: 2021-01-18; 网络版发表日期: 2021-03-02 \\ 国家重点研发计划(编号: 2019YFC0408302)和江苏省重点研发计划社会发展项目(编号: BE2018702)资助
}

\begin{abstract}
摘要水解-沉淀是无机金属盐能够发挥混凝作用的关键过程. 金属盐的价态、浓度、配体浓度以及水的 $\mathrm{pH}$ 均 对混凝性能有着重要影响. 为准确对比各种无机金属盐作为混凝剂的优劣势和适用范围, 本文通过热力学平衡常 数的计算, 并与混凝实验相结合, 解析和验证了铝、铁、钛和锆4种金属的无机盐在有/无配体情况下的沉淀区域 图和水合/水解物种的形态分布, 定量描述了多核水解产物在4种金属盐水解-沉淀过程中的贡献, 并从溶液化学的 角度揭示了水中常见配体影响混凝性能的内在机制。上述结果为无机金属盐混凝剂的研发和应用提供了理论基 础, 对于准确理解混凝性能和机制具有重要意义.
\end{abstract}

关键词铝盐, 铁盐, 钛盐, 锆盐, 混凝, 配体, 水处理

\section{1 引言}

混凝是水处理工艺中最基础的单元之一，主要用 于浊度物质(胶体颗粒以及悬浮物)的去除. 混凝包括 凝聚和絮凝两个过程. 其中, 凝聚过程主要取决于无 机金属盐的水解缩聚性能, 而絮凝过程主要由水解产 物的表面电荷和分形维数决定. 因此, 了解无机金属 盐的水解-沉淀过程, 对于准确理解混凝性能和机制具 有重要意义.

铝盐和铁盐是目前应用最为广泛的无机盐混凝 剂 ${ }^{[1 ~ 3]}$, 而钛盐和锆盐由于电荷高、水解速率快, 是具 有很大应用潜力的混凝剂 ${ }^{[4,5]}$. 自 20 世纪 50 年代以来,
铝盐和铁盐的水溶液化学得到了长足的发展 ${ }^{[6]}$. 有关 铝盐和铁盐的水解缩聚过程, 已有大量文献报道. 然 而，在不同的报道中，金属盐的水解形态分布存在较 大差异, 原因包括: (1) 所采用的金属盐的水解以及沉 淀溶解平衡常数不同 ${ }^{[7]}$; (2) 有些形态分析只考虑了单 核的情况，而金属盐在水解过程中会形成多核羟基聚 合物; (3) 在混凝过程中, 原位形成的氢氧化物均为无 定形态, 部分研究在计算水解和沉淀溶解平衡时, 采用 的是晶态(氢)氧化物, 而非无定形态氢氧化物的溶度 积常数 ${ }^{[8]}$. 从热力学数据来看, 固体氢氧化物 $\left(\mathrm{M}(\mathrm{OH})_{n}\right.$ $(\mathrm{s}))$ 与无定形态氢氧化物 $\left(\mathrm{M}(\mathrm{OH})_{n}(\mathrm{am})\right)$ 在溶解-沉淀平 衡常数上存在较大差异 ${ }^{[7]}$.

引用格式: Gan Y, Zhang L, Wu B, Zheng H, Zhang S. Analysis of key factors in the coagulation of metal salts based on the calculation of hydrolysis-precipitation distribution. Sci Sin Chim, 2021, 51: 458-467, doi: 10.1360/SSC-2020-0221 
如图1所示, 由于在计算时未考虑多核羟基水解产 物(图1(a))或采用了固体氢氧化物 $\left(\mathrm{M}(\mathrm{OH})_{n}(\mathrm{~s})\right)$, 而非无 定形沉淀 $\left(\mathrm{M}(\mathrm{OH})_{n}(\mathrm{am})\right)$ 的平衡常数(图1(b)), 来自两篇 高引论文的沉淀区域图缺失了氢氧化物在固-液两相 界面间的平衡, 导致了形态分析的偏差, 进而影响到对 金属盐水解沉淀过程及混凝出水中金属残留的准确分 析. 例如, 与实际铝盐和铁盐混凝过程相比, 图 1中 $\mathrm{Al}(\mathrm{OH})_{3}$ 与 $\mathrm{Fe}(\mathrm{OH})_{3}$ 的沉淀区域偏宽，总溶解态金属浓 度偏低. 因此，采用校正后的平衡常数并考虑多核水 解物种，对金属盐离子的水解沉淀过程进行重新分析 是非常有必要的.

配体对金属盐的水解具有重要影响. 水中存在各 种各样的无机配体(氟化物、磷酸盐和硫酸盐等)和有 机配体(氨羧配体和羟羧配体等)，这些配体通过与金 属盐离子形成金属配合物而抑制其水解，或者通过影 响水解产物的电荷分布影响絮凝过程，导致混凝性能 恶化. 例如, 当存在 $1.0 \mathrm{mmol} / \mathrm{L}$ 氟化钠时，由于铝氟间 强的配合作用, 导致无定形 $\mathrm{Al}(\mathrm{OH})_{3}$ 的可溶解部分提高 了2 3 个数量级 ${ }^{[10]}$; 当柠檬酸与 $\mathrm{Fe}_{2}\left(\mathrm{SO}_{4}\right)_{3}$ 的摩尔比为 0.05 时, 铁盐混凝过程无絮体生成 ${ }^{[11]}$. 目前对金属盐混 凝过程中的配体影响尚缺乏全面的对比研究. 明晰配 体对混凝过程的影响有助于篮选和开发具有抗配体负 荷冲击性能的混凝剂.

基于此, 本研究首先通过文献调研, 根据热力学判 据, 篮选出了铝、铁、钛和锆 4 种金属盐在标准状态下 可靠的水解、沉淀溶解和配位平衡常数; 然后借助 Matlab和Microsoft Excel软件分析了4种金属盐的水 解-沉淀过程以及配体对金属盐物种形态的影响; 最后 用实际混凝实验验证了计算结果的准确性，揭示了配 体影响金属盐混凝性能的内在机制. 本文结果可为金 属盐混凝剂的开发、应用以及混凝机制解析提供理论 指导.
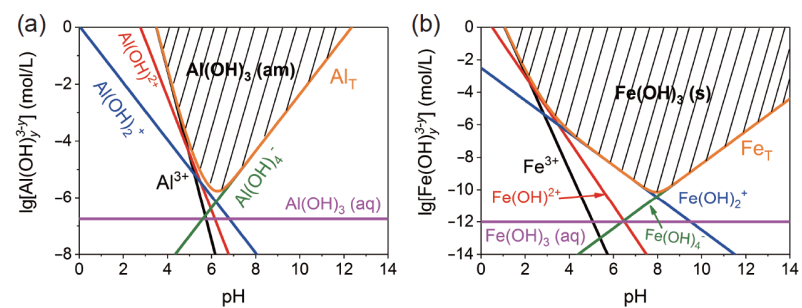

图 $1 \mathrm{Al}^{3+}$ (a)和 $\mathrm{Fe}^{3+}(\mathrm{b})$ 的沉淀区域图 ${ }^{[1,9]}$ (网络版彩图) Figure 1 Precipitate charts of (a) $\mathrm{Al}^{3+}$ and (b) $\mathrm{Fe}^{3+}$ [1,9] (color online).

\section{2 实验部分}

\section{1 试剂与仪器}

氯化钛 $\left(\mathrm{TiCl}_{4}\right)$ 和氯化锆 $\left(\mathrm{ZrCl}_{4}\right)$ (分析纯, 上海阿拉 丁生化科技股份有限公司，中国); 氯化铁 $\left(\mathrm{FeCl}_{3}\right)$ 、氯 化铝 $\left(\mathrm{AlCl}_{3}\right)$ 、磷酸二氢钾 $\left(\mathrm{KH}_{2} \mathrm{PO}_{4}\right)$ 、乙二胺四乙酸二 钠盐(EDTA-2Na)、盐酸 $(\mathrm{HCl})$ (分析纯, 国药集团化学 试剂有限公司，中国); 氟化钠 $(\mathrm{NaF})$ 和硫酸钠 $\left(\mathrm{Na}_{2} \mathrm{SO}_{4}\right)$ (分析纯，上海麦克林生化科技有限公司，中国); 高岭 土(6000目，上海跃江钛白化工制品有限公司，中国); 腐殖酸纳 $(45 \%$ 70\%, Acros Organics, 赛默飞世尔科技 有限公司, 美国); 氢氧化钠 $(\mathrm{NaOH})$ (分析纯, 南京化学 试剂股份有限公司, 中国).

自动控制混凝六联摚拌仪(ZR4-6, 深圳市中润水 工业技术发展有限公司, 中国); pH计(FE28, 梅特勒公 司, 瑞士); 浊度计(2100N, 哈希公司, 美国); 电感耦合 等离子体发射光谱仪(iCAP7400, 赛默飞世尔科技有限 公司, 美国); Zeta电位分析仪(Nano ZS90, 马尔文公司, 英国); 紫外分光光度计(UV2700, 岛津公司, 日本).

\section{2 实验方法}

\subsection{1 计算模拟}

根据近些年对金属离子水解-沉淀过程的最新研 究进展, 从文献或美国国家标准与技术研究院 $(\mathrm{Na}-$ tional Institute of Standards and Technology, NIST)数 据库篎选出用于计算水解 - 沉淀过程的平衡常 数 ${ }^{[6,7,12 \sim 19]}$, 借助Matlab软件(R2019a, MathWorks公司, 美国)求解非齐次线性方程组得到不同形态金属物种 的含量随 $\mathrm{pH}$ 变化的分布曲线 ${ }^{[4]}$. 在氢氧化物沉淀溶解 平衡的分析过程中, 根据水解平衡常数和溶度积常数 $($ 式 $(1,2))$, 计算出各物种浓度与 $\mathrm{pH}$ 的关系, 其中 $\mathrm{M}^{z^{+}}$代 表 $\mathrm{Al}^{3+} 、 \mathrm{Fe}^{3+} 、 \mathrm{Ti}^{4+}$ 和 $\mathrm{Zr}^{4+} ; z$ 为金属离子的化合价; $K_{\mathrm{Sam}}$ 为无定形氢氧化物的溶度积常数; $K_{\mathrm{w}}$ 为水的电离平衡 常数; $\beta_{x, y}$ 为金属离子的水解平衡常数. 在计算配体影 响时，金属盐浓度均为 $1.0 \mathrm{mmol} / \mathrm{L}$. 配体的浓度为 $0.1 \sim 10 \mathrm{mmol} / \mathrm{L}$ (详见网络版补充材料文本 $\mathrm{S} 1$ 和文本 $\mathrm{S} 2$ ). 所选取水解平衡常数的合理性，通过模拟计算与 实验结果的吻合度进行了验证:

$$
\begin{aligned}
& {\left[\mathrm{M}^{z+}\right]=K_{\mathrm{Sam}} K_{\mathrm{w}}^{-z}\left[\mathrm{H}^{+}\right]^{z}} \\
& {\left[\mathrm{M}_{x}(\mathrm{OH})_{y}^{z x-y}\right]=K_{\mathrm{Sam}}^{x} K_{\mathrm{w}}^{-z x} \beta_{x, y}\left[\mathrm{H}^{+}\right]^{z x-y}}
\end{aligned}
$$




\subsection{2 混凝实验}

使用ZR4-6型自动控制六联混凝仪开展混凝烧杯 实验. 实验所用水样为HA-Kaolin模拟水, 主要水质参 数如下: $\mathrm{pH} \quad 8.1 \pm 0.1$, 水温 $20^{\circ} \mathrm{C}$, 浊度 $48 \pm 2 \mathrm{NTU}$, $\mathrm{UV}_{254}=0.100 \pm 0.005 \mathrm{~cm}^{-1}$. 在探究不同配体的影响时, 上述模拟水样中添加一定量的配体 $\left(\mathrm{KH}_{2} \mathrm{PO}_{4} 、 \mathrm{NaF}\right.$ 、 $\mathrm{Na}_{2} \mathrm{SO}_{4}$ 或EDTA-2Na)并调节初始pH至8.1左右. 无机盐 混凝剂储备液浓度为 $0.1 \mathrm{~mol} / \mathrm{L}$, 混凝剂优化投加量为 $0.2 \mathrm{mmol} / \mathrm{L}$, 混凝后出水 $\mathrm{pH}$ 在6.5 7.3 (图S1, 网络版补 充材料). 混凝程序如下: 模拟水样预先混合快速搅拌 (200 r/min) $1 \mathrm{~min}$ 后投加指定量的混凝剂，在该条件下 继续快速摚拌 $1 \mathrm{~min}$ 以实现充分的凝聚过程, 随后转入 时长为 $15 \mathrm{~min}$ 的慢速搅拌 $(40 \mathrm{r} / \mathrm{min})$ 絮凝阶段, 在快速 搅拌结束后取样分析絮体的Zeta电位变化; 慢摚结束 后静置沉淀 $20 \mathrm{~min}$, 取上层清液测定残留浊度; 上层清 液经 $0.45 \mu \mathrm{m}$ 聚醚砜水性滤膜过滤后测定 $U V_{254}$ 以及残 留金属浓度.

\section{3 结果与讨论}

\section{1 金属盐水解-沉淀分析}

\subsection{1 单核体系}

金属离子在水中以水合离子 $\mathrm{M}\left(\mathrm{H}_{2} \mathrm{O}\right)_{n}{ }^{2+}$ 的形态存 在. 水分子与金属中心的配位属于路易斯酸碱反应, 电 荷由水转移到金属，这使得质子从配位的水中解离比 从非配位的水中更容易。由金属水合离子形成金属氢 氧化物的过程即称为水解(式(3)). 以 $\mathrm{Fe}^{3+}$ 为例, 水解过 程可以表述为式(4).

$$
\begin{aligned}
& \mathrm{M}\left(\mathrm{H}_{2} \mathrm{O}\right)^{z+} \stackrel{-\mathrm{H}^{+}}{\longrightarrow} \mathrm{MOH}^{(z-1)^{+}} \stackrel{-\mathrm{H}^{+}}{\longrightarrow} \mathrm{MO}^{(z-2)^{+}} \\
& \mathrm{Fe}\left(\mathrm{H}_{2} \mathrm{O}\right)_{6}^{3+} \stackrel{-\mathrm{H}^{+}}{\longrightarrow} \mathrm{Fe}\left(\mathrm{H}_{2} \mathrm{O}\right)_{5} \mathrm{OH}^{2+} \stackrel{-\mathrm{H}^{+}}{\longrightarrow} \mathrm{Fe}\left(\mathrm{H}_{2} \mathrm{O}\right)_{4}(\mathrm{OH})_{2}^{+} \\
& \stackrel{-\mathrm{H}^{+}}{\longrightarrow} \mathrm{Fe}\left(\mathrm{H}_{2} \mathrm{O}\right)_{3}(\mathrm{OH})_{3} \stackrel{-\mathrm{H}^{+}}{\longrightarrow} \mathrm{Fe}\left(\mathrm{H}_{2} \mathrm{O}\right)_{2}(\mathrm{OH})_{4}^{-} \stackrel{-\mathrm{H}^{+}}{\longrightarrow}
\end{aligned}
$$$$
\mathrm{Fe}\left(\mathrm{H}_{2} \mathrm{O}\right)(\mathrm{OH})_{5}^{2-} \stackrel{-\mathrm{H}^{+}}{\longrightarrow} \mathrm{Fe}(\mathrm{OH})_{6}^{3-}
$$

金属的水解产物主要由金属离子 $\mathrm{M}^{2+}$ 与三种氧配 体 $\left(\mathrm{H}_{2} \mathrm{O} 、 \mathrm{OH}^{-}\right.$和 $\left.\mathrm{O}^{2-}\right)$ 组成. 根据质子的离解能力, 水解 过程主要受 $\mathrm{H}^{+}$浓度及金属电荷密度的影响. 较低的溶 液 $\mathrm{pH}$ (高 $\mathrm{H}^{+}$浓度)和较高的金属电荷密度(低电荷 $z$ 、高 配位数 $n$ 和存在强供电子效应的配体等)都会抑制金属 离子的水解过程，从而减少氢氧化物的生成. 如图2所

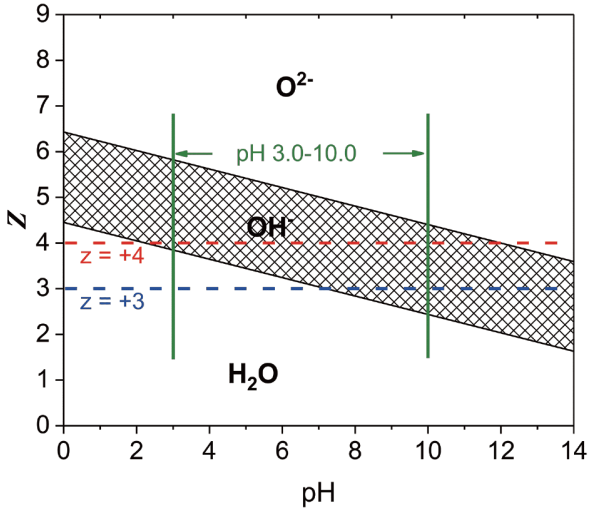

图 2 不同价态(z)金属的水解/水合区域图(网络版彩图) Figure 2 The hydrolysis/hydration of metal ions (Z: valence) (color online).

示, 一般情况下, 当 $z<4$ 时, 金属离子仅能产生水合物和 羟基配合物; 当 $z>4$ 时，金属离子可以形成羟基以及氧 配合物; 而当 $z=4$ 时可以形成水合物、羟基和氧的多种 配合物 ${ }^{[20]}$. 因此, 相比于 $\mathrm{Al}^{3+}$ 和 $\mathrm{Fe}^{3+}, \mathrm{Ti}^{4+}$ 和 $\mathrm{Zr}^{4+}$ 能在更 低的 $\mathrm{pH}$ 条件下水解形成羟基配合物. 钛盐和锆盐的水 解平衡常数较铁盐和铝盐高出 $8 \sim 16$ 个数量级(表 $S 1$, 网 络版补充材料), 表明钛盐和锆盐更容易混凝沉淀.

需要注意的是, 已有大量实验证明在酸性区域 $\mathrm{Ti}(\mathrm{IV})$ 主要以 $\mathrm{TiO}^{2+}$ 形式, 而不是以 $\mathrm{Ti}^{4+}$ 或 $\mathrm{Ti}(\mathrm{OH})^{2+}$ 形式 存在 ${ }^{[7]}$. 计算表明, 选用 $\mathrm{Ti}^{4+}$ 或 $\mathrm{TiO}^{2+}$ 作为起始物种对沉 淀区域的影响并不大(图 $\mathrm{S} 2$ ), $\mathrm{TiO}^{2+}$ 浓度变化与 $\mathrm{Ti}^{4+}$ 体系 中的 $\mathrm{Ti}(\mathrm{OH})_{2}{ }^{2+}$ 类似. 因此, 在后续的分析过程中, 主要 以 $\mathrm{TiO}^{2+}$ 作为钛盐水解-沉淀的起始组分.

根据水解和沉淀溶解平衡常数(表 $\mathrm{S} 1$ 和 $\mathrm{S} 2$ ) 计算可 绘制4种金属氢氧化物的沉淀区域图，结果如图3(a)所 示. 若仅考虑单核体系，当金属盐浓度为 $1.0 \mathrm{mmol} / \mathrm{L}$ 时, 钛盐(1.3 14.0)和锆盐(0.2 14.0)的沉淀 $\mathrm{pH}$ 区域更 宽(表 $\mathrm{S} 3$ ), 而 $\mathrm{Al}^{3+}$ 对应的氢氧化物沉淀的形成条件最为 苛刻(5.5 9.5). 另外, 从氢氧化物的沉淀溶解平衡来看, 在整个 $\mathrm{pH}$ 范围内, $\mathrm{Al}(\mathrm{OH})_{3}$ 的溶解度最高. 当 $\mathrm{pH}<4.0$ 时, $\mathrm{Fe}(\mathrm{OH})_{3}$ 的溶解度明显高于 $\mathrm{TiO}(\mathrm{OH})_{2}$ 和 $\mathrm{Zr}(\mathrm{OH})_{4}$. 4 种金 属的沉淀 $\mathrm{pH}$ 范围顺序依次为: $\mathrm{Zr}(\mathrm{OH})_{4}>\mathrm{TiO}(\mathrm{OH})_{2}>$ $\mathrm{Fe}(\mathrm{OH})_{3}>\mathrm{Al}(\mathrm{OH})_{3}$. 因此, 对于混凝出水残余金属浓度 要求较高的处理工艺可以选择钛盐或锆盐混凝剂, 尤 其在偏酸性条件下.

\subsection{2 多核体系}

在金属水解形成氢氧化物沉淀的过程中，存在缩 

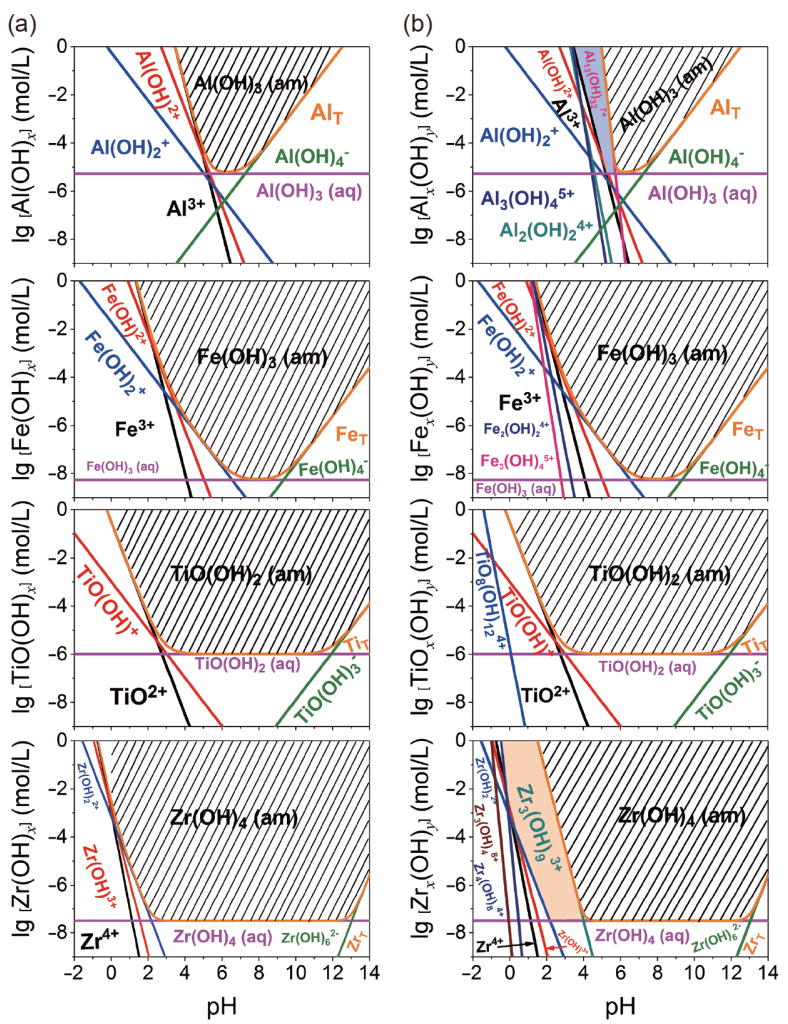

图 34 种金属盐的沉淀区域图. (a) 只考虑单核水解物; (b) 同时考虑多核水解物(网络版彩图)

Figure 3 Precipitation chart of the four metal salts. (a) Mononuclear system; (b) polynuclear system (color online).

聚过程, 即形成多核水解产物. 缩聚反应包括亲核加成 (式(5))和亲核取代(式(6)). 由于水配体中的氧原子不 具有亲核性质, 方程中的 $\mathrm{OX}$ 只可能为 $\mathrm{O}^{2-}$ 或 $\mathrm{OH}^{-}$. 此 外, 含 $\mathrm{O}^{2-}$ 基团的水解物很难参与缩聚反应, 式(5)中的 $\mathrm{OY}$ 为 $\mathrm{OH}^{-}$或 $\mathrm{H}_{2} \mathrm{O}$. 因此, 单核物种只有在含有羟基配 合物时才可以发生缩合反应 ${ }^{[20]}$. 由于四价离子比三价 离子存在更宽的羟基配合物区域(图2), 相比于铝盐和 铁盐, 钛盐和锆盐单核水解产物更倾向于缩聚形成多 核水解产物.

$$
\begin{aligned}
& \mathrm{M}-\mathrm{OX}+\mathrm{M}-\mathrm{OY} \rightarrow \mathrm{M}-\mathrm{O}(\mathrm{X})-\mathrm{M}+\mathrm{OY} \\
& \mathrm{M}-\mathrm{OX}+\mathrm{M}^{z^{+}} \rightarrow \mathrm{M}-\mathrm{O}(\mathrm{X})-\mathrm{M}
\end{aligned}
$$

基于Ekberg和Brown对所有金属水解缩聚数据的 热力学分析以及温度、离子强度的校正 ${ }^{[7]}$, 本文将以 下多核产物, 包括 $\mathrm{Al}_{2}(\mathrm{OH})_{2}{ }^{4+} 、 \mathrm{Al}_{3}(\mathrm{OH})_{4}{ }^{5+} 、 \mathrm{Al}_{13}(\mathrm{OH})_{32}{ }^{7+}$ 、 $\mathrm{Fe}_{2}(\mathrm{OH})_{2}{ }^{4+} 、 \mathrm{Fe}_{3}(\mathrm{OH})_{4}{ }^{5+} 、(\mathrm{TiO})_{8}(\mathrm{OH})_{12}{ }^{4+} 、 \mathrm{Zr}_{3}(\mathrm{OH})_{4}{ }^{8+}$ 、 $\mathrm{Zr}_{3}(\mathrm{OH})_{9}{ }^{3+}$ 和 $\mathrm{Zr}_{4}(\mathrm{OH})_{8}{ }^{8+}$ 纳入水解和沉淀溶解平衡的计
算. 如图3所示, 是否考虑多核水解物, 对铝和锆的沉淀 区域有着显著影响, 且具有明显的浓度效应(图S3和 S4). 随着金属盐浓度的增大, 多核水解物占比逐渐增 大. 其中, $\mathrm{Al}_{13}(\mathrm{OH})_{32}{ }^{7+}$ 和 $\mathrm{Zr}_{3}(\mathrm{OH})_{9}{ }^{3+}$ 分别具有较宽的 $\mathrm{pH}$ 主导区域，而铁和钛的多核水解物即使在高浓度水平 下占比也不足 $5 \%$.

由此可知, 铝盐和锆盐的单核水解产物会通过缩 聚产生大量带正电荷的多核物种, 影响氢氧化物沉淀 的形成. 对于混凝过程, 较高的正电荷水解产物可以 发挥更强的电中和作用, 从而脱稳胶体. 但是由于过 高的正电荷会引发电荷反转, 必须严格控制 $\mathrm{pH}$ 及剂量 条件以防止发生重稳. 这与文献中报道的铝盐和锆盐 易发生重稳现象比较吻合 ${ }^{[5,21]}$. 由于过快的缩聚, 钛盐 在酸性条件下才显示出较强的电中和能力, 因此在中 碱性条件下钛盐混凝过程主要由网捕卷扫机制主 导 ${ }^{[22]}$.

\section{2 水解形态与沉淀区域的准确度分析}

为了定量描述平衡计算的准确与否对水解形态与 沉淀溶解区域的影响, 在只考虑单核水解物的前提下, 本文计算了铝盐和铁盐在有、无沉淀生成条件(金属 总浓度为 $0.1 \sim 10 \mathrm{mmol} / \mathrm{L}$ )下的形态分布, 并与文献报 道的数据做了对比 ${ }^{[1,9]}$.

无论是铝盐还是铁盐，本文所绘制的形态分布图 与文献图存在较大差异. 如图4所示, 当无固体物种生 成时, 即使不考虑多核水解物, 因为选取的平衡常数不 同, 文献图中的溶解态 $\mathrm{Al}(\mathrm{OH})_{3}(\mathrm{aq})$ 在 $\mathrm{pH}$ 5.0 8.0区间的 分布分数比本文计算得到的分布分数低 1 2 个数量级, 而溶解态 $\mathrm{Fe}(\mathrm{OH})_{3}(\mathrm{aq})$ 在 $\mathrm{pH} \quad 8.0$ 时的浓度更是低了 2 个 数量级. 当金属盐浓度处于常见的混凝剂使用浓度区 间时 $(0.01 \sim 1.0 \mathrm{mmol} / \mathrm{L})$, 文献计算得到的溶解态铝浓 度明显偏低, 而无定型固体 $\mathrm{Fe}(\mathrm{OH})_{3}(\mathrm{am})$ 形成的 $\mathrm{pH}$ 区 间偏宽. 这些不同说明平衡常数的选取会在很大程度 上影响金属盐水解形态的分布区间，导致在评估混凝 剂投加量、适用 $\mathrm{pH}$ 以及混凝出水残留金属浓度时出 现很大偏差.

混凝出水中金属的残留量以及形态分布会影响后 续处理单元的处理效率. 混凝和膜滤的联用是当前及 今后一段时期内水处理领域发展的重点 ${ }^{[23,24]}$. 残留金 属氢氧化物会对膜造成不可逆的污染 ${ }^{[25,26]}$. 因此，准 确计算和了解金属氢氧化物在混凝区域的浓度对于工 


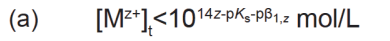

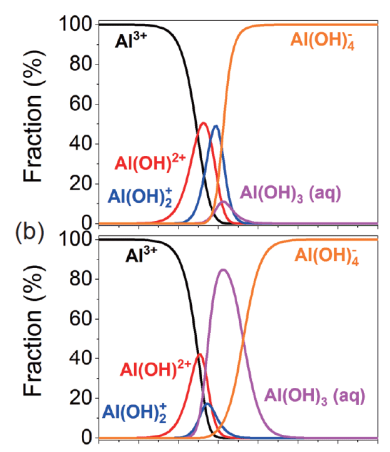

(1)
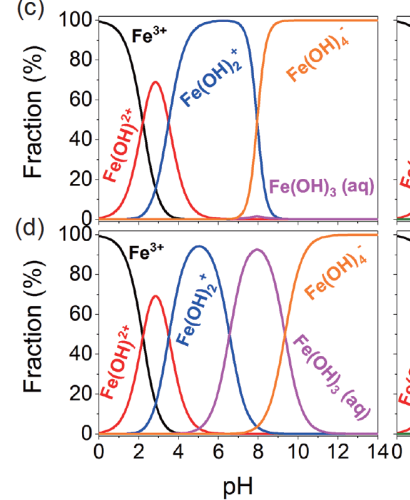

$\left[\mathrm{M}^{\mathrm{z+}}\right]_{\mathrm{t}}=1.0 \times 10^{-5} \mathrm{~mol} / \mathrm{L}$
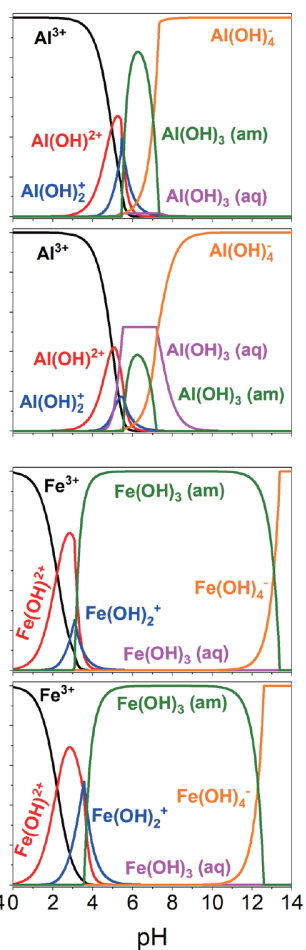
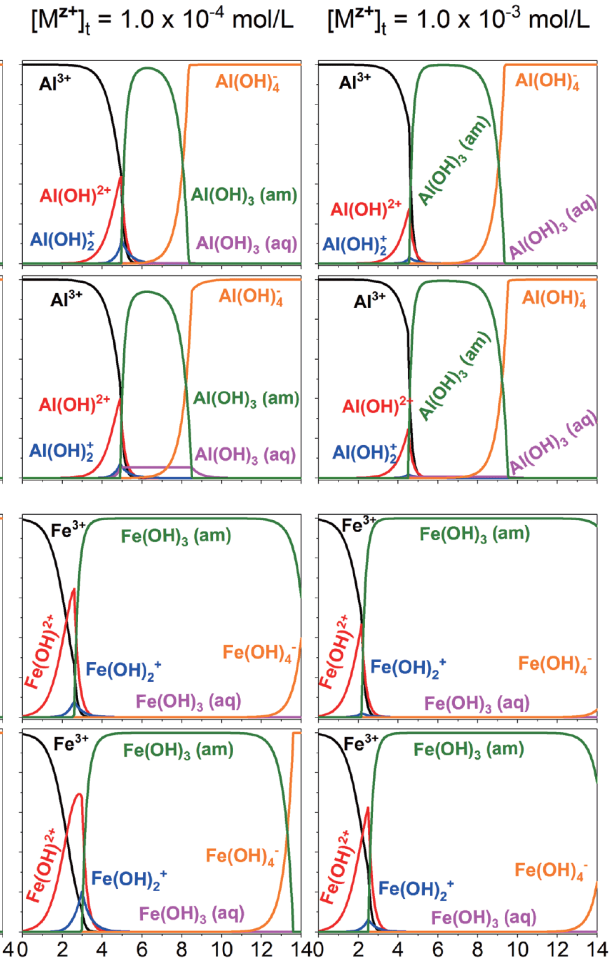

$\mathrm{pH}$

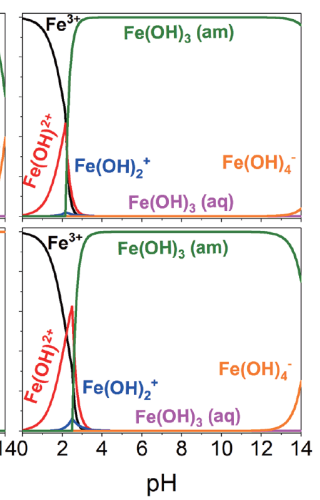

图 4 铝盐和铁盐的水解形态分布: (a, c) 文献 ${ }^{[1,9]}$ 结果; (b, d) 本文的计算结果(网络版彩图)

Figure 4 Hydrolysis speciation of $\mathrm{Al}$ and $\mathrm{Fe}$ salts: (a, c) from literature [1,9]; (b, d) this study (color online).

艺的设计至关重要. 本文通过实际混凝实验验证了计 算结果的可靠性. 如图S1(d)所示, 4种金属盐混凝出水 中金属残留量的实测值和理论计算值相吻合. 当氯化 铝投加量为 $0.01 \sim 0.10 \mathrm{mmol} / \mathrm{L}$ 时 $(\mathrm{pH} \quad 7.1 \sim 7.8$ ), 混凝出 水残留铝浓度为 $0.1191 \sim 0.1674 \mathrm{mg} / \mathrm{L}$, 且明显高于其他 三种金属盐, 这与本文计算结果相吻合.

与无机离子和有机物的配位是金属离子的重要归 趋途径. 在混凝过程中共存污染物对金属盐的水解、 沉淀及絮凝均存在一定影响. 因此, 在实际混凝处理 过程中, 需要综合考虑水质因子对混凝沉淀的影响. 以下重点分析了各种配体对金属盐水解-沉淀以及混 凝行为的影响.

\section{3 配体对金属盐水解-沉淀的影响}

金属盐的水解建立在与水分子配位的基础上，其 他配体会通过占据水分子的配位点抑制金属离子的水 解，或者通过与金属离子反应生成溶解性配合物或不 溶性沉淀. 本文根据金属-配体间的配合稳定常数和配 体的酸解离平衡常数等(表S4 S6), 计算了水中典型的
3种无机配体(氟化物、正磷酸盐和硫酸盐)与有机配 体(EDTA)在环境浓度下对4种金属盐水解-沉淀过程 的影响, 进而评估配体对金属盐混凝过程的影响.

\subsection{1 氟化物}

氟化物是水体中常见的污染物, 饮用水中规定氟 浓度不得超过 $1.0 \mathrm{mg} / \mathrm{L}$, 然而在一些地下水中氟浓度 会高于此限值. 在电镀废水和矿山废水等工业废水中 氟浓度甚至高达几百上千 $\mathrm{mg} / \mathrm{L}$. 混凝沉淀是处理含氟 废水的主要技术之一, 氟化物通过配位、吸附和共沉 淀等方式同金属氢氧化物一起从水中分离 ${ }^{[5]}$. 铝盐和 锆盐具有优异的除氟性能; 而钛盐和铁盐除氟性能较 差, 仅在偏酸性条件下起作用 ${ }^{[4]}$. 从金属盐的水解过程 分析，根据 $\mathrm{F}^{-}$和 $\mathrm{OH}^{-}$配体与金属离子的配位常数评估 氟化物对 4 种金属盐水解的相对影响程度(表S5). 氟化 物通过配位作用抑制金属水解排序: $\mathrm{Al}^{3+}>\mathrm{Zr}^{4+}>\mathrm{TiO}^{2+}$ $>\mathrm{Fe}^{3+}$, 这也从侧面证明了金属盐通过与氟配位形成配 合物进而除氟的途径.

氟化物对金属水解-沉淀过程的影响与其浓度关 
系很大(图5(a)). 当金属盐浓度为 $0.01 \sim 0.1 \mathrm{mmol} / \mathrm{L}$, 氟 化物处于低浓度时 $(0.1 \mathrm{mmol} / \mathrm{L})$ 时, $\mathrm{Al}^{3+}$ 水解体系中在 $\mathrm{pH}$ 为5.0 8.0 的区间内均存在一定量的铝氟配合物 $\left(\mathrm{AlF}_{2}{ }^{+} 、 \mathrm{AlF}_{3}\right.$ 和 $\left.\mathrm{AlF}_{4}{ }^{-}\right)$, 而 $\mathrm{Zr}^{4+}$ 水解体系受 $\mathrm{F}^{-}$配位影响 并不显著，这与我们前期锆盐和铝盐混凝除氟相关研 究结果一致 ${ }^{[5]}$. 当氟化物处于高浓度时 $(10 \mathrm{mmol} / \mathrm{L}), 4$ 种金属盐均在较宽的 $\mathrm{pH}$ 范围内形成大量氟配合物. 其 中, 铝盐水解被完全抑制, 在 $\mathrm{pH}<8.0$ 的区间内均为铝氟 配合物; 铁盐水解受影响程度最低，在正常起作用 $\mathrm{pH}$ 区间(6.0 9.0)几乎不受影响; 钛盐和锆盐结果类似, 仅 在酸性条件下受明显抑制, $\mathrm{TiO}(\mathrm{OH})_{2}$ 和 $\mathrm{Zr}(\mathrm{OH})_{4}$ 仍可以 在较宽的 $\mathrm{pH}$ 范围内形成.

金属离子的加入也在很大程度上影响了氟化物的 存在形态 $^{[4]}$. 通过分析氟化物形态的变化可以更好地 理解金属盐混凝除氟的机制. 对于低氟水混凝处理过 程，锆盐主要先形成具有表面羟基的氢氧化锆，然后 再通过吸附、离子交换和氢键等作用去除氟化物; 然 而其他三种金属主要先形成氟配合物，再通过共沉淀
去除．当存在中高浓度的氟化物时，含氟配合物的占 比明显升高，含氟配合物的不充分水解会导致混凝出 水中残留金属浓度升高，进而增加出水的健康风险. 基于上述分析，可优选锆盐为处理低氟水样的混凝剂, 而铁盐可作为处理高氟水样的混凝剂.

\subsection{2 正磷酸盐}

磷酸盐广泛存在于天然水体 $(0.001 \sim 0.3 \mathrm{mg} / \mathrm{L})$ 、 生活污水(4.0 7.0 mg/L) 以及工业废水(可高达几千 $\mathrm{mg} / \mathrm{L}$ ) 中, 其主要的存在形式为正磷酸盐. 铁盐和铝盐可与磷 酸盐发生化学沉淀作用，因此它们被广泛地用作除磷 混凝剂 ${ }^{[27]}$.

4种金属离子与各种形态的正磷酸盐离子的配合 稳定常数如表 $\mathrm{S} 5$ 所示, 其中 $\mathrm{Al}^{3+} 、 \mathrm{Fe}^{3+} 、 \mathrm{TiO}^{2+}$ 和 $\mathrm{HPO}_{4}{ }^{2-}$ (中碱性 $\mathrm{pH}$ 下正磷酸盐的主要形态)的反应平 衡常数分别为 $10^{7.4} 、 10^{8.3}$ 和 $10^{4.48}$, 这表明正磷酸盐与 铁、铝盐的亲和能力强于钛盐. 另外，从金属正磷酸 盐的溶度积来看，在较宽的 $\mathrm{pH}$ 范围内均可生成

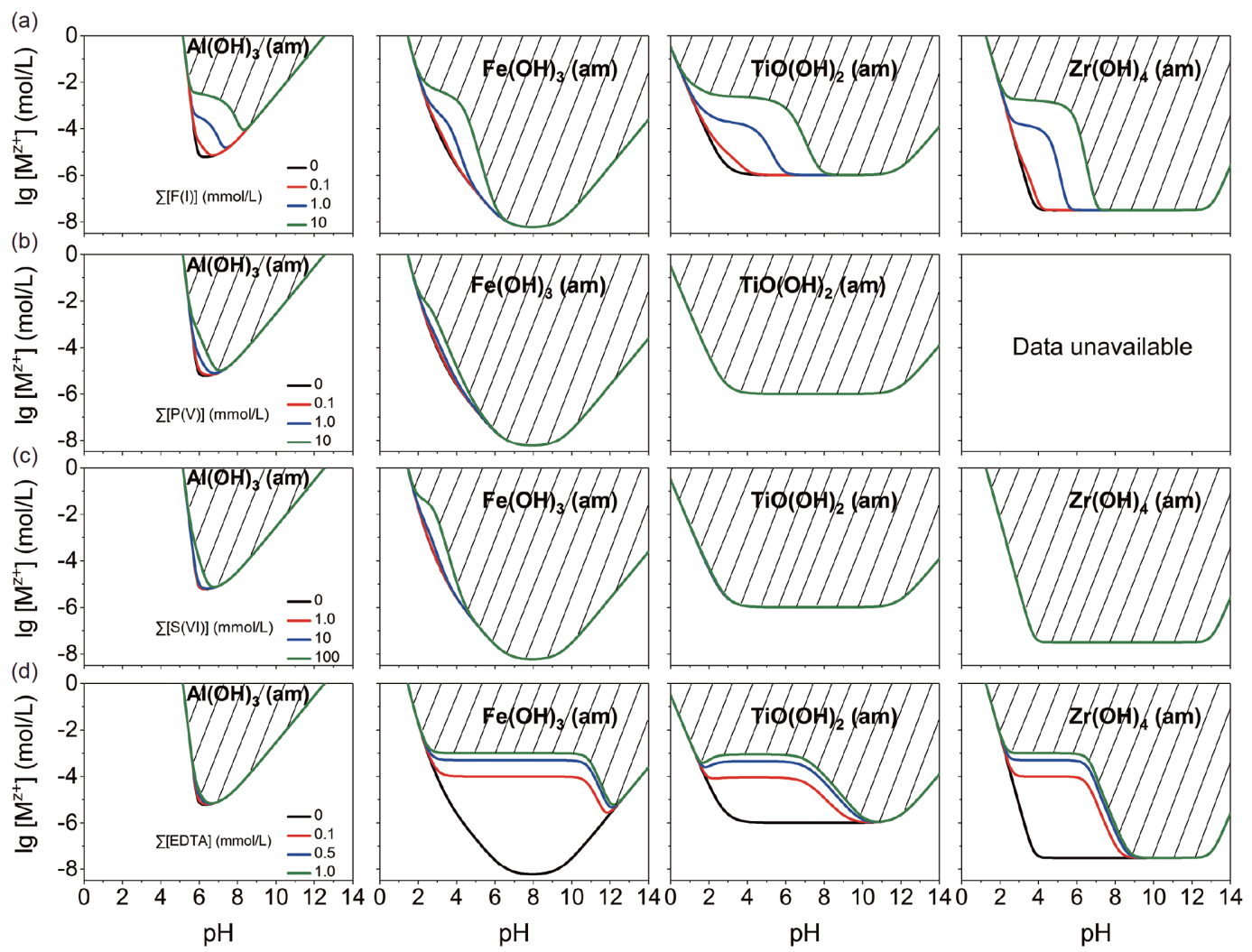

图 5 配体对金属盐沉淀区域的影响. (a) 氟化物; (b) 磷酸盐; (c) 硫酸盐; (d) EDTA (网络版彩图)

Figure 5 Effects of ligands on the precipitation zones of the four metal salts. (a) Fluoride; (b) phosphate; (c) sulfate; (d) EDTA (color online). 
$\mathrm{Zr}_{3}\left(\mathrm{PO}_{4}\right)_{4}$, 但无 $\mathrm{TiOHPO}_{4}$ 沉淀生成.

如图5(b)所示，正磷酸盐对三种金属水解的影响 程度如下: $\mathrm{Al}^{3+}>\mathrm{Fe}^{3+}>\mathrm{TiO}^{2+}$. 尽管正磷酸盐与铁盐的配 位能力强于 $\mathrm{Al}^{3+}$ ，但 $\mathrm{Al}^{3+}$ 的水解速率明显慢于 $\mathrm{Fe}^{3+}$, 这 导致在 $0.1 \sim 10 \mathrm{mmol} / \mathrm{L}$ 的正磷酸盐存在下，铝离子的水 解受到了更大的抑制. 当正磷酸盐浓度为 $0.1 \mathrm{mmol} / \mathrm{L}$ 时铁铝盐沉淀区域变化较小, $\mathrm{AlHPO}_{4}{ }^{+} 、 \mathrm{AlH}_{2} \mathrm{PO}_{4}{ }^{2+}$ 、 $\mathrm{FeHPO}_{4}{ }^{+}$和 $\mathrm{FeH}_{2} \mathrm{PO}_{4}{ }^{2+}$ 均可忽略不计. 随着正磷酸盐浓 度增大, $\mathrm{Al}^{3+}$ 和 $\mathrm{Fe}^{3+}$ 分别在 $\mathrm{pH}$ 5.0 7.0和2.0 5.0酸性区 域的水解行为受到一定抑制. 然而, 高浓度正磷酸盐对 $\mathrm{TiO}^{2+}$ 的水解仍然可以忽略不计(图5(b)).

\subsection{3 硫酸盐}

硫酸盐是水体中常见的阴离子之一，在高盐废水 中浓度可达上万 $\mathrm{mg} / \mathrm{L}$. 根据表S5中的配位常数，相比 于氟化物，硫酸盐属于弱配体. 低浓度 $(1.0 \mathrm{mmol} / \mathrm{L})$ 的 硫酸盐对4种金属的水解-沉淀基本不产生影响(图5 (c)). 当硫酸盐浓度为 $100 \mathrm{mmol} / \mathrm{L}$ 时, $\mathrm{Al}^{3+}$ 和 $\mathrm{Fe}^{3+}$ 的沉淀 过程由于金属硫酸盐配合物的形成分别在 $\mathrm{pH} \quad 5.0 \sim 7.0$ 和2.0 5.0受到轻微抑制, 而 $\mathrm{TiO}^{2+}$ 和 $\mathrm{Zr}^{4+}$ 仍不受影响. 这表明钛盐和锆盐受硫酸盐配体影响的 $\mathrm{pH}$ 范围窄且 程度更低。尽管在常规混凝 $\mathrm{pH} \quad(6.0 \sim 9.0)$ 和剂量 (0.01 1.0 mmol/L) 范围内，金属氢氧化物的形成并未 受到显著影响, 但硫酸盐配合物 (如 $\mathrm{Al}\left(\mathrm{SO}_{4}\right)_{2}{ }^{-}$和 $\mathrm{Fe}\left(\mathrm{SO}_{4}\right)_{2}{ }^{-}$等 $)$会导致正电荷水解物种的降低.

\subsubsection{EDTA}

混凝可以有效去除一些大分子疏水性有机物，而 对于小分子亲水有机物去除较差. 研究表明，水体中 的小分子有机配体是造成混凝性能变差和出水残余金 属升高的重要因素 ${ }^{[11,28]}$. 一些含有羧基和羟基等配位 基团的有机物(如EDTA、柠檬酸和草酸等)可以与金 属离子发生配位作用形成金属有机配合物，这不仅抑 制了金属氢氧化物的形成，而且加速了金属离子的迁 移过程. 本文选取典型的小分子有机物EDTA作为有 机配体, 来探究有机配体对金属盐水解过程的影响.

根据NIST提供的配位数据(表S6), 分别计算了 4种 金属离子在不同EDTA浓度下的沉淀区域，结果如图5 (d) 所示. EDTA对铝盐水解-沉淀影响最小, 但是对 铁、钛和锆盐的影响非常大, 排序如下: $\mathrm{Fe}^{3+}>\mathrm{TiO}^{2+}$ $>\mathrm{Zr}^{4+}>>\mathrm{Al}^{3+}$. 随着EDTA的浓度从 0.1 增至 $1.0 \mathrm{mmol} / \mathrm{L}$,
铁、钛和锆三种金属盐与EDTA形成的金属有机配合 物也可以相应地从 0.1 增至 $1.0 \mathrm{mmol} / \mathrm{L}$. 当EDTA $\left(\mathrm{H}_{4} \mathrm{~L}\right)$ 为 $1.0 \mathrm{mmol} / \mathrm{L}$ ，金属盐浓度为 $0.1 \mathrm{mmol} / \mathrm{L}$ 时，由于 $\mathrm{FeL}^{-} 、 \mathrm{Fe}(\mathrm{OH}) \mathrm{L}^{2-} 、[(\mathrm{FeOH}) \mathrm{L}]_{2}{ }^{4-} 、 \mathrm{TiOL}^{2-} 、 \mathrm{ZrL}$ 、 $\mathrm{Zr}(\mathrm{OH}) \mathrm{L}^{-}$和 $[\mathrm{Zr}(\mathrm{OH}) \mathrm{L}]_{2}{ }^{2-}$ 等有机配合物的形成, 以上三 种金属盐的水解都受到显著抑制, 开始沉淀 $\mathrm{pH}$ 分别由 3.0、1.8和 2.6 提高到 $11.4 、 8.1$ 和7.1 (表 S3)，这表明铁 盐受EDTA的影响区间更大，而钛盐和锆盐可通过提 高pH来缓解EDTA的配合作用.

\section{4 配体对金属盐混凝过程的影响}

为验证计算模拟的结果，本文以典型HA-高岭土 模拟废水为处理对象，评价了不同浓度配体对 4 种金 属盐混凝性能的影响. 在优化投加量 $0.2 \mathrm{mmol} / \mathrm{L}$ 下, 4 种混凝剂混凝出水浊度去除率 $>90 \%, \mathrm{UV}_{254}$ 去除率 $>65 \%\left(\mathrm{ZrCl}_{4}<\mathrm{TiCl}_{4}<\mathrm{FeCl}_{3}<\mathrm{AlCl}_{3}\right)$, 残留金属浓度 $<$ $0.04 \mathrm{mg} / \mathrm{L} \quad\left(\mathrm{TiCl}_{4}<\mathrm{FeCl}_{3}<\mathrm{ZrCl}_{4}<\mathrm{AlCl}_{3}\right)$, 出水 $\mathrm{pH}$ 在 6.5 7.3之间(图S1). 当引入无机或有机配体后，4种金 属盐的混凝性能发生不同的变化(图6(a c)). 配体通过 抑制金属盐的水解过程以及改变水解产物的表面电荷 来影响混凝性能.

氟对4种金属盐除浊性能、除UV $\mathrm{V}_{254}$ 性能和残留金 属浓度的影响与金属-氟之间配位能力具有一定的相 关性, 说明氟化物主要通过配位作用影响混凝性能(表 S5和图6(b)). 在高氟浓度且中性条件下, 铝盐、钛盐和 锆盐与氟化物形成的配合物抑制了金属的水解过程, 铁盐几乎不受影响(图5). 与空白对照相比, 由氟离子所 导致的4种金属盐混凝絮体的Zeta电位下降顺序如下: $\mathrm{Al}^{3+}(23.0 \mathrm{mV})>\mathrm{Zr}^{4+}(12.2 \mathrm{mV})>\mathrm{TiO}^{2+}(6.5 \mathrm{mV})>\mathrm{Fe}^{3+}$ $(4.6 \mathrm{mV})$ (图6(d)). 这表明氟化物存在下, 铝盐和锆盐 絮凝过程受到更大的抑制。当氟化物浓度为 $1.0 \mathrm{mmol} / \mathrm{L}$ 时，残留铝和锆浓度分别为 1.650 和 $0.063 \mathrm{mg} / \mathrm{L}$ ，而铁和钛无残留; 当氟化物浓度增至 $10 \mathrm{mmol} / \mathrm{L}$ 时，残留铝、钛和锆分别为 $3.33 、 0.30$ 和 $0.57 \mathrm{mg} / \mathrm{L}$, 而铁仍然几乎无残留(图6(c)).

正磷酸盐配体基本不影响除浊效果且混凝后几乎 无金属残留(图6(c)), 但是高浓度正磷酸盐会导致絮体 变细, 沉降更慢. 当正磷酸盐浓度升高至 $10 \mathrm{mmol} / \mathrm{L}$, 铝 盐混凝出水浊度由 $1.2 \mathrm{NTU}$ 增至3.0 NTU, 这可能归因 于微弱的 $\mathrm{AlHPO}_{4}{ }^{+}$的形成，而其他三种金属盐残留浊 度并未升高(图6(c)). 这表明在中性条件下, 4种金属盐 
(a)
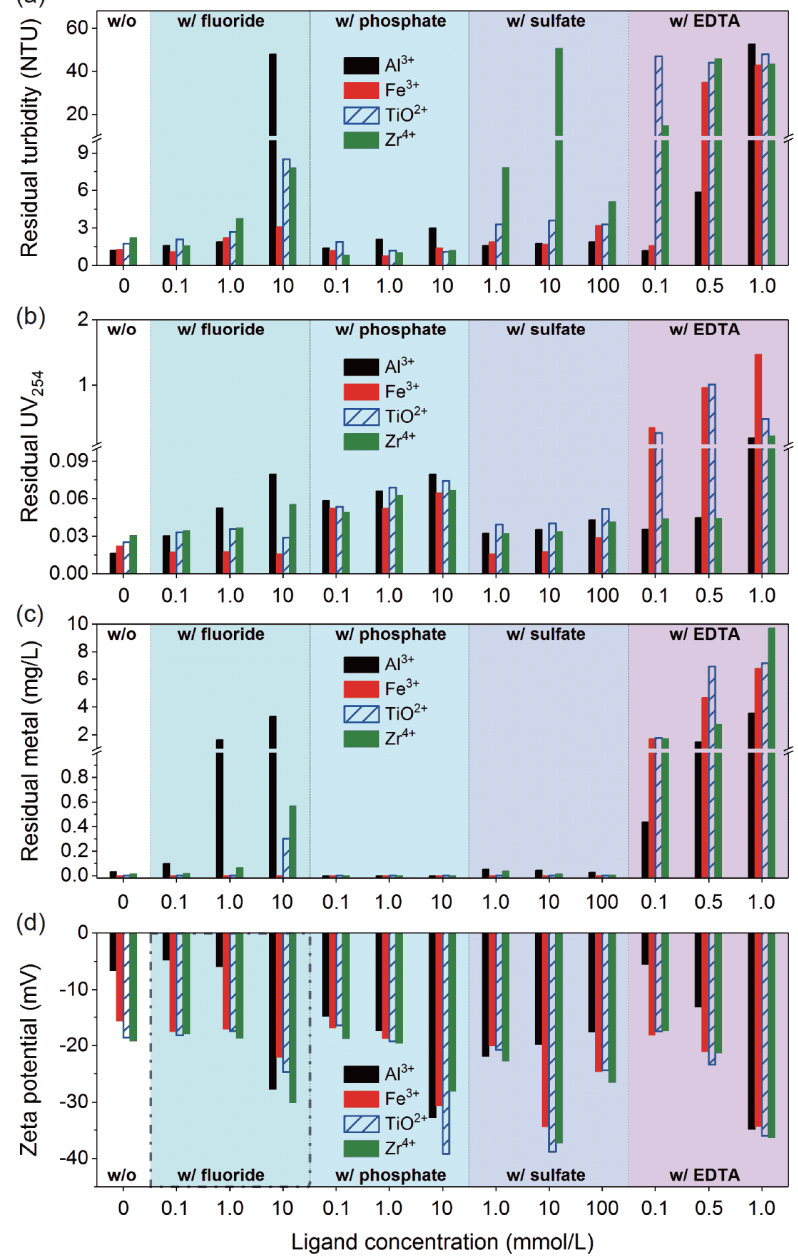

图 6 配体对金属盐 $\left(\left[\mathrm{M}^{z+}\right]_{\mathrm{t}}: 0.2 \mathrm{mmol} / \mathrm{L}\right)$ 混凝去除水溶液浊 度 $(\mathrm{a})$ 、有机物 (以UV 254 表示) $(\mathrm{b})$ 、残留金属浓度 $(\mathrm{c})$ 和絮体 Zeta电位(d)的影响(网络版彩图)

Figure 6 Effects of ligands on the removal of (a) turbidity, (b) organics (expressed with $\mathrm{UV}_{254}$ ), (c) residual metal concentration, and (d) Zeta potential by the four metal salts $\left(\left[\mathrm{M}^{z+}\right]_{\mathrm{t}}: 0.2 \mathrm{mmol} / \mathrm{L}\right)($ color online).

混凝剂的水解过程几乎不受正磷酸盐配体的影响，但 是由于正磷酸盐吸附在水解产物表面，会影响后续的 絮凝过程. 如图6(d)所示, 高浓度正磷酸盐使得絮体表 面Zeta电位变得更负(从-19 -7 mV降至-39 -28 $\mathrm{mV})$ ，更大的静电排斥会导致絮体生长变慢. 另一方 面，由于正磷酸盐竞争了用于吸附HA的大量吸附位 点, 因此UV 254 的去除率随着正磷酸盐浓度的增大而大 幅降低(图6(b)).

由残留金属浓度以及Zeta电位变化可以看出(图6 (d))，在中性条件下硫酸盐几乎不影响金属的水解过
程，然而会通过改变水解物的电荷影响絮凝过程，特 别是对于铁、铝和钛体系. 当硫酸盐浓度为 $10 \mathrm{mmol} / \mathrm{L}$ 时，铁、铝和钛盐所生成的絮体表面的电 荷下降了约 $20 \mathrm{mV}$. 锆盐混凝除浊性能受影响程度最 为显著, 这也说明絮凝过程对于锆混凝的重要性 ${ }^{[21]}$.

在4种配体中，EDTA对4种金属盐混凝性能的影 响最大(图6). 对于除浊效率: $\mathrm{Al}^{3+}>\mathrm{Fe}^{3+}>\mathrm{Zr}^{4+}>\mathrm{TiO}^{2+}$, 当 EDTA浓度大于 $0.1 \mathrm{mmol} / \mathrm{L}$ 时, 铁、钛和锆盐均无明显 絮体生成; 对于 $\mathrm{UV}_{254}$ 的去除而言, $\mathrm{Zr}^{4+}$ 和 $\mathrm{Al}^{3+}$ 在EDTA 浓度为 $0.1 \sim 0.5 \mathrm{mmol} / \mathrm{L}$ 影响较小, 而 $\mathrm{TiO}^{2+}$ 和 $\mathrm{Fe}^{3+}$ 与 EDTA形成配合物在 $254 \mathrm{~nm}$ 处产生更高的吸光度. 此 外, 强配位能力的EDTA会导致混凝出水中残留金属 浓度剧增以及Zeta电位降低(图6(d)). 因此, EDTA在很 大程度上影响了金属盐凝聚与絮凝两个过程，特别是 对于钛盐和锆盐混凝剂.

由配体存在下4种金属盐的混凝结果来看, 配体不 仅影响金属的水解过程, 还影响缩聚、吸附及絮凝过 程. 氟化物由于配位作用会使铝盐、钛盐和锆盐水解 过程受阻; 磷酸盐及硫酸盐会使水解物的Zeta电位变 得更负而影响絮凝过程; 具有强螯合能力的EDTA使 得混凝性能严重恶化, 特别是对于钛盐和锆盐混凝剂. 从混凝机制、混凝性能和抗配体影响三个方面综合来 看(图7)，4种金属盐的混凝属性由强到弱分别为: $\mathrm{Ti}^{4+}$ $>\mathrm{Fe}^{3+}>\mathrm{Al}^{3+} \& \mathrm{Zr}^{4+}$.

综上所述，有机配体比无机配体对混凝效果的影 响更为显著. 计算模拟可以对金属水解过程进行精确 分析，但对后续的进一步缩聚、吸附和絮凝等过程无 法做出完全准确的判断.
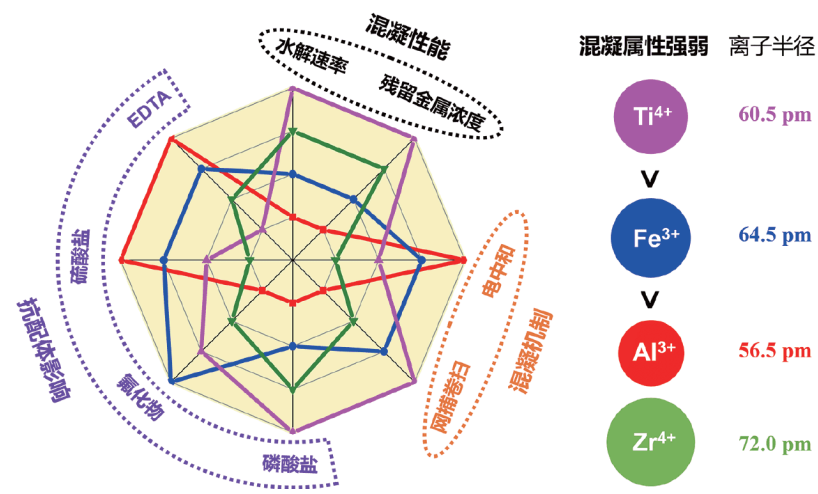

图 74 种金属盐混凝属性雷达图(网络版彩图)

Figure 7 Radar chart of coagulation properties of the four metal salts (color online). 


\section{4 结论}

本文通过模拟计算分析了4种金属盐的水解-沉淀 区域. 钛盐和锆盐形成氢氧化物沉淀的 $\mathrm{pH}$ 区域宽于传 统的铝盐和铁盐. 从沉淀-溶解平衡看, 在偏酸性条件 下 (pH 3.0 4.0时), 溶解态钛盐和锆盐的浓度比铁盐低 1 2个数量级, 而此时铝盐基本以溶解态形式存在. 因 此, 钛盐和锆盐混凝剂具有适用 $\mathrm{pH}$ 范围宽和残留金属 浓度低的优点. 多核水解产物在铝和锆盐混凝体系中 有较大占比, 对钛和铁盐的影响很小. 因此, 对于铝盐 和锆盐，在沉淀溶解分析中忽略多核物种将会有较大 偏差.

几种常见配体对金属盐水解过程的影响如下. (1) 有机配体对4种金属盐的水解具有显著抑制, 其中铝盐 和铁盐的受影响 $\mathrm{pH}$ 区间及程度大于钛盐和锆盐. (2) 氟化物通过配位作用抑制金属水解，其中铝盐的水解 由于铝氟配合物的形成而受很大影响，铁盐几乎不受 氟化物影响，而钛盐和锆盐仅在酸性条件下受明显抑
制.（3）正磷酸盐对铝盐和铁盐的水解抑制能力远强 于对钛盐的抑制; 在较宽的 $\mathrm{pH}$ 范围内均可生成 $\mathrm{Zr}_{3}\left(\mathrm{PO}_{4}\right)_{4}$, 但无 $\mathrm{TiOHPO}_{4}$ 沉淀生成. (4) 弱配体硫酸盐 对金属水解过程影响最小. 因此, 在混凝过程中可以通 过复配使用来发挥各自优势, 弥补短板.

在低温低浊水/酸性废水处理、深度除氟以及混 凝-膜滤等工艺流程中，以钛盐和锆盐作为替代混凝 剂, 有望解决铝盐和铁盐混凝中存在的一些问题, 如 水解动力学受限、絮体特性差、残留金属给后续处理 单元带来负面影响等. 因此, 近 10 年来, 钛盐和锆盐混 凝剂受到了大量的关注，大量新型的无机高分子聚钛 以及聚锆混凝剂被开发出来．在由低分子向高分子， 单一到复合的药剂研发中, 需要准确了解金属盐的水 解-沉淀过程. 本研究通过对比分析 4 种典型金属盐的 水解-沉淀区域，深入探讨了金属盐混凝过程中配体的 影响, 不但丰富了有关金属盐混凝沉淀的溶液化学, 且 为新型混凝剂的开发及应用提供了理论指导.

\section{补充材料}

本文的补充材料见网络版http://chemen.scichina.com. 补充材料为作者提供的原始数据, 作者对其学术质量和内容负责.

\section{参考文献}

1 Duan J, Gregory J. Adv Colloid Interface Sci, 2003, 100-102: 475-502

2 Rajala K, Grönfors O, Hesampour M, Mikola A. Water Res, 2020, 183: 116045

3 Skaf DW, Punzi VL, Rolle JT, Kleinberg KA. Chem Eng J, 2021, 386: 123807

4 Gan Y, Li J, Zhang L, Wu B, Huang W, Li H, Zhang S. Chem Eng J, 2020, 406: 126837

5 Gan Y, Wang X, Zhang L, Wu B, Zhang G, Zhang S. Chemosphere, 2019, 218: 860-868

6 Tang HX. Inorganic Polymer Flocculation Theory and Flocculants. Beijing: China Architecture \& Building Press, 2006 (in Chinese) [汤鸿宵. 无 机高分子絮凝理论与絮凝剂. 北京: 中国建筑工业出版社, 2006]

7 Brown PL, Ekberg C. Hydrolysis of Metal Ions. New York: John Wiley \& Sons, 2016

8 Ratnaweera H. Multidisciplinary Advances in Efficient Separation Processes. Washington: American Chemical Society, 2020

9 Tchobanoglous G. Wastewater Engineering: Treatment and Resource Recovery. 5th ed. New York: McGraw-Hill, 2014

10 Yu WZ, Gregory J, Graham N. Environ Sci Technol, 2016, 50: 1828-1833

11 Liang L, Yu WZ, Xu L. Acta Sci Circum, 2013, 33: 2428-2431 (in Chinese) [梁亮, 俞文正, 徐砧. 环境科学学报, 2013, 33: 2428-2431]

12 Einaga H, Komatsu Y. J Inorg Nucl Chem, 1981, 43: 2443-2448

13 ciavatta L, Ferri D, Riccio G. Polyhedron, 1985, 4: 15-22

14 Buettner KM, Valentine AM. Chem Rev, 2012, 112: 1863-1881

15 Speight JG. Lange's Handbook of Chemistry. 16th ed. New York: McGraw-Hill, 2005

16 Lagno F, Demopoulos GP. Environ Tech, 2006, 27: 1217-1224

17 Smith RM, Martell AE. Critical Stability Constants Inorganic Complexes. New York: Plenum Press, 1976

18 Goldstein G. Anal Chem, 1964, 36: 243-244 
Liu YJ, Xu H, Jiao RY, Duan SX, Wang DS, Duan JM, Yang Y, Liu N. China Water Wastewater, 2019, 5: 55-59 (in Chinese) [刘艳静, 徐慧, 焦 茹媛, 段淑璇, 王东升, 段晋明, 杨杨, 刘娜. 中国给水排水, 2019, 5: 55-59]

\title{
Analysis of key factors in the coagulation of metal salts based on the calculation of hydrolysis-precipitation distribution
}

\author{
Yonghai Gan, Li Zhang, Bingdang Wu, Hongcen Zheng, Shujuan Zhang* \\ State Key Laboratory of Pollution Control and Resource Reuse, School of the Environment, Nanjing University, Nanjing 210023, China \\ *Corresponding author (email: sjzhang@nju.edu.cn)
}

\begin{abstract}
Hydrolysis and precipitation are the key processes that determine the coagulation performance of inorganic metal salts. The valence and concentration of metal salts, ligands, and solution $\mathrm{pH}$ all influence the coagulation performance. In order to get a full picture on the pros and cons of various inorganic metal salts as coagulants, this paper conducted thermodynamic equilibrium calculations and coagulation experiments to analyze and verify the hydrolysisprecipitation areas and speciation of four inorganic metal salts (aluminum, iron, titanium, and zirconium) in the absence and presence of ligands. In addition, the contributions of polynuclear hydrolysates in the hydrolysis and precipitation of the four metal ions were quantitatively described. Furthermore, the underlying mechanisms of the effects of common aquatic ligands on coagulation performance were discussed from the perspective of solution chemistry. The above results provide a theoretical basis for the development and application of inorganic metal salt coagulants, and are of great significance for understanding the coagulation performance and mechanisms accurately.
\end{abstract}

Keywords: aluminum salt, ferric salt, titanium salt, zirconium salt, coagulation, ligand, water treatment

doi: $10.1360 /$ SSC-2020-0221 